Reprod. Nutr. Dévelop., 1988, 28 Suppl. n¹, 19-32

\title{
Données nouvelles sur les bactéries cellulolytiques du rumen
}

G. FONTY (1) (2), Evelyne FORANO (1), Geneviève GAUDET (1) (3), Sylvie KOMISARCZUCK (4), Ph. GOUET (1)

(1) Laboratoire de Microbiologie, I.N.R.A Theix, 63122 Ceyrat, France.

(2) Laboratoire de Biologie Comparée des Protistes, C.N.R.S. UA 138, Université Clermont-Ferrand II. 63170 Aubière, France.

(3) Laboratoire de Microbiologie, Université Clermont-Ferrand II, 63170 Aubière, France.

(4) Station de Recherches sur la Nutrition des Herbivores Unité de la Digestion Microbienne, I.N.R.A. Theix, 63122 Ceyrat, France.

\section{Introduction.}

L'isolement, la purification et l'identification des espèces bactériennes cellulolytiques du rumen actuellement connues ont commencé vers 1950 après que Hungate eut mis au point une technique fiable pour cultiver les espèces bactériennes extrêmement sensibles à l'oxygène.

Au cours des années cinquante, les caractères morphologiques, métaboliques, fermentaires, les besoins nutritionnels, les types de cellulose attaquée, les milieux de culture ont été décrits pour: Bacteroides succinogenes, (B. succinogenes), actuellement nommé Fibrobacter succinogenes (Montgomery, Flesher et Stahl, 1988), Ruminococcus albus (R. albus), Ruminococcus flavefaciens (R. flavefaciens), Butyrivibrio fibrisolvens (B. fibrisolvens) et quelques autres moins répandues. Le phénomène d'adhérence des cellules bactériennes aux fibres ainsi que les complexes hydrolasiques ont aussi été observés ou analysés à plusieurs reprises. Ces résultats ont déjà fait l'objet de revues (Hungate, 1966 ; Bryant, 1973).

Au cours de ces dix dernières années, le perfectionnement des techniques biochimiques, et l'utilisation de la biologie moléculaire ont sensiblement amélioré nos connaissances de la physiologie de ces espèces, de leurs complexes hydrolasiques, des mécanismes de genèse et de conservation de l'énergie ainsi que de l'adhésion. Par ailleurs la maîtrise de l'élevage des agneaux gnotobiotiques a montré tout son intérêt pour étudier l'écologie des populations et leurs activités spécifiques in vivo. Enfin le comportement des espèces cellulolytiques vis-à-vis de l'introduction d'additifs alimentaires antimicrobiens dans les rations et de l'apparition de techniques nouvelles de complémentation et de conditionnement des fourrages a été à l'origine d'observations originales. Cette revue a pour objet de faire le point des connaissances acquises dans ces domaines au cours des dernières années. 


\section{Implantation chez le préruminant.}

Les espèces bactériennes cellulolytiques apparaissent rapidement après la naissance aussi bien chez le veau (Anderson et al., 1987) que chez l'agneau (Fonty et al., 1987a). Dès l'âge de 4 jours, les trois quarts des agneaux élevés en troupeau en hébergent. Tous en sont pourvus à la fin de la première semaine, avant la consommation d'aliment solide. La population atteint alors un niveau proche de celui observé chez les adultes $\left(10^{7}-10^{8}\right.$ cellules $\mathrm{ml}^{-1}$ de contenu de rumen). La cellulose n'est nullement indispensable à la présence des espèces cellulolytiques dans le rumen puisqu'elles se maintiennent à un niveau relativement élevé $\left(10^{6}\right.$ cellules $\left.\mathrm{ml}^{-1}\right)$ chez des agneaux nourris exclusivement avec du lait de vache. Par contre leur transmission est conditionnée par les contacts étroits entre le nouveau-né et sa mère ou d'autres ruminants (Fonty et al., 1987a).

La présence d'une microflore non cellulolytique diversifiée et complexe est indispensable aux espèces cellulolytiques pour coloniser le rumen en leur procurant des composés indispensables à leur croissance ou en détruisant des substances inhibitrices présentes dans le contenu de rumen (Fonty et al., 1983). II est en effet très difficile, sinon impossible, d'implanter ces espèces chez des agneaux gnotoxéniques lorsque la microflore est trop simplifiée, même si celle-ci est composée des espèces dominantes chez l'adulte et choisies de manière à assurer théoriquement les principales fonctions de dégradation des aliments du rumen (Mann et Stewart, 1974 ; Lysons et al., 1976 ; Hobson, Mann et Stewart, 1981). Ainsi $B$. succinogenes ne peut s'établir chez des agneaux dont la flore est réduite à 16 souches (Fonty et al., 1988a). Son implantation à un niveau comparable à celui observé chez des animaux conventionnels $\left(10^{8}-10^{9}\right.$ bact. $\left.\mathrm{ml}^{-1}\right)$ a toutefois été obtenue chez des agneaux gnotoxéniques préalablement inoculés avec 180 souches d'espèces non cellulolytiques isolées du rumen de jeunes agneaux conventionnels. Enfin, la colonisation après ensemencement par des espèces bactériennes cellulolytiques a pu être facilement obtenue chez des agneaux nés conventionnellement puis placés dans un environnement aseptique quelques jours après la naissance (Gouet et al., 1984 ; Fonty et al., 1988b).

\section{Activité des différentes espèces in vitro et in vivo.}

La capacité des trois principales espèces (B. succinogenes, $R$. albus, $R$. flavefaciens) à dégrader in vitro différents substrats cellulosiques a déjà fait l'objet de nombreux travaux. La plupart ont montré que B. succinogenes était l'espèce la plus active en culture pure. Elle est capable de dégrader très efficacement les fibres de coton et la cellulose hautement cristalline (Stewart, 1981). Toutefois cette activité diffère dans de larges proportions selon les souches (Morris et Van Gylswyk, 1980 ; Graham et al., 1985 ; Chesson et al., 1986).

Les bactéries cellulolytiques peuvent solubiliser à la fois la cellulose et les hémicelluloses de fragments végétaux (Morris et Van Gylswyk, 1980 ; Graham et 
al., 1985 ; Chesson et al., 1986). Les Ruminococcus utilisent en général à la fois les pentoses et les hexoses alors que la plupart des souches de $B$. succinogenes ne métabolisent pas les pentoses résultant de l'hydrolyse des hémicelluloses (Morris et Van Gylswyk, 1980; Graham et al., 1985). Les Ruminococcus solubilisent une quantité plus importante d'arabinoxylanes de la paille que de cellulose, alors que $B$. succinogenes solubilise ces deux constituants en quantités égales (Graham et al., 1985). Le traitement des pailles à l'ammoniac favorise leur dégradation ainsi que la croissance des bactéries cellulolytiques (Kolankaya et al., 1985).

L'efficacité d'une seule espèce, mesurée in vivo chez l'agneau gnotobiotique vis-à-vis de différents substrats cellulosiques (Fonty et al., 1987b, 1988b), peut être aussi importante, voire plus, que celle d'une association d'espèces. En outre l'activité de chacune dépend du substrat. Ainsi $R$. flavefaciens FD1 dégrade plus efficacement la cellulose de ray-grass que $B$. succinogenes, ce dernier étant plus efficace vis-à-vis de la cellulose de la paille traitée ou non à l'ammoniac. La quantité de cellulose de paille dégradée par B. succinogenes a été supérieure à celle mesurée chez des agneaux conventionnels et elle n'a pas augmenté lorsque $B$. succinogenes a été associé à $R$. flavefaciens (Fonty et al., 1987b).

\section{Adhérence.}

L'adhérence des bactéries cellulolytiques à la cellulose et aux fibres végétales, première étape de la cellulolyse, est un phénomène observé de longue date (Hungate, 1950). Il a fait l'objet de nombreuses études descriptives lors du développement de la microscopie électronique (voir revue de Cheng et Costerton, 1980 ; Cheng et al., 1984) mais les mécanismes de l'adhérence n'ont pas encore été clairement élucidés. Ainsi l'existence de structures spécifiques de la bactérie permettant son adhérence à des récepteurs végétaux n'a pas encore été montrée. Au cours de ces dernières années les recherches ont été focalisées essentiellement sur le rôle des facteurs physicochimiques (Kudo, Cheng et Costerton, 1987; Fonty, Roger et Gouet, 1987c ; Morris, 1987 ; Roger et al., 1988). L'effet de ces facteurs varie selon les espèces ce qui suggère l'existence de mécanismes d'adhérence différents. Ainsi la température agit sur l'adhérence de $B$. succinogenes S85 et l'oxygène l'inhibe fortement alors que celle de $R$. flavefaciens 007 n'est nullement influencée par ces deux paramètres. En revanche l'adhérence de ce dernier à la cellulose Avicel est fortement diminuée en présence de méthyl et d'hydroxyéthyl cellulose alors que celle de $B$. succinogenes est peu affectée (Roger et al., 1988). L'adhérence de $R$. albus à la cellulose Whatman CF 11 est fortement inhibée par la carboxyméthylcellulose et la méthylcellulose mais elle est résistante à l'action des détergents, de l'EDTA et du thiocyanate de potassium (Morris, 1987).

\section{Activités enzymatiques.}

Les cellulases.

Trois familles d'enzymes collaborent à la dégradation de la cellulose. 
- Les endoglucanases souvent appelées CMCase (carboxyméthyl cellulase) ou HECase (hydroxyéthyl cellulase) coupent les liaisons glycosidiques en $\beta$-1-4 à l'intérieur de l'homopolymère de glucose.

- Les exoglucanases libèrent du cellobiose (cellobiohydrolases ou cellobiosidases ou cellodextrinases) ou du glucose (glucohydrolases) à partir de l'extrémité non réductrice des polymères de glucose. biose.

- Les $\beta$-glucosidases ou cellobiases hydrolysent spécifiquement le cello-

\section{Endoglucanases.}

Ces enzymes, les plus étudiées, sont généralement présentes dans le milieu de culture, mais également liées aux membranes.

Chez $R$. albus (Wood, Wilson et Stewart, 1982) plusieurs protéines de poids moléculaire variant de $5.10^{4}$ à $1,5.10^{6} \mathrm{~d}$, possédant une activité CMCase sont présentes dans le milieu de culture. Les cinétiques comparées de dégradation de la CMC par viscosimétrie et par dosage des sucres réducteurs libérés indiquent une nature commune à ces enzymes. Des protéines enzymatiques de bas poids moléculaire pourraient exister sous forme de complexes stables de haut poids moléculaire dans la paroi des bactéries et sous forme libre ou plus ou moins associée dans le milieu de culture.

Les enzymes liés à la paroi peuvent être facilement relargués par lavages répétés avec du tampon. Les bactéries apparaissent lors de l'observation en microscopie à balayage, couvertes de protubérances qui sont éliminées par ces mêmes lavages dans du tampon. Elles sont communes à de nombreuses bactéries cellulolytiques et peuvent être associées à l'activité cellulolytique (Lamed et al., 1987).

Chez $R$. albus un gène codant pour une activité spécifiquement CMCase a été cloné. Les caractéristiques de l'enzyme issu de ce clonage sont peu différentes de celles d'une CMCase parallèlement purifiée à partir de R. albus (Kawai et al., 1987).

L'activité CMCase chez $R$. flavefaciens a été retrouvée codée par des gènes différents donc portée par des protéines distinctes (Barros et Thomson, 1987) et pour certains des clones obtenus associée à des activités cellobiohydrolase et $\beta$-glucosidase (Huang et al., 1987).

Une pluralité enzymatique est également observée chez B. succinogenes (Groleau et Forsberg, 1983a, b; Schellhorn et Forsberg, 1984). A partir d'une culture sur cellulose l'activité endoglucanase se répartit entre la fraction extracellulaire : $80 \%$, et les bactéries : $20 \%$. Sept composants enzymatiques ont été globalement identifiés et comparés, cinq sont plutôt des endoglucanases existant sous forme associée dans des fragments membranaires en suspension dans le milieu, deux autres composants sont plutôt des exoglucanases (Schellhorn et Forsberg, 1984).

L'activité endoglucanase associée aux cellules chez $B$. succinogenes est retrouvée associée à la membrane externe (Gaudet, 1987a, b) et dans l'espace 
périplasmique des bactéries en phase exponentielle de croissance sur glucose ou sur cellobiose; les techniques de chocs osmotiques utilisées pour préparer les extraits périplasmiques ne permettent ni d'exclure l'origine membranaire de cette activité ni une localisation périplasmique transitoire avant fixation dans la membrane externe.

Les fractions membranaires possédant une activité endoglucanase, retrouvées dans le surnageant de culture sont assimilées aux vésicules membranaires très caractéristiques formées par la bactérie. Ces structures naissent dans des poches entre le substrat cellulosique et la bactérie, mais elles ne semblent pas avoir un rôle fondamental dans la cellulolyse. En effet, elles sont absentes des cultures jeunes sur cellulose alors que les nombreuses divisions, et les réserves polysaccharidiques des cellules indiquent le bon fonctionnement de celles-ci et par conséquent de la cellulolyse (Gaudet et Gaillard, 1987).

Un gène codant pour une triple activité endoglucanase, cellobiohydrolase et surtout lichenase a été cloné à partir de B. succinogenes (Taylor et al., 1987). Entre l'enzyme cloné et l'activité enzymatique mesurée chez $B$. succinogenes des différences importantes ont été constatées : profils électrophorétiques différents, activité spécifique inférieure, stimulation par le calcium, répression par le glucose pour l'enzyme cloné, absence de stimulation par le calcium, et de répression par le glucose chez $B$. succinogenes. Toutes ces différences suggèrent que le gène cloné ne code que pour un enzyme mineur du complexe cellulasique.

Cavicchioli et Watson (1987) ont également cloné chez $E$. coli un gène codant pour une activité endoglucanase à partir d'une autre souche de $B$. succinogenes (souche-S-O). L'expression de cette endoglucanase n'est par contre réprimée ni par le glucose ni par le cellobiose.

\section{Exoglucanase.}

Chez R. albus, après $50 \mathrm{~h}$ de culture sur cellulose, une cellobiohydrolase extracellulaire est active sur para nitrophenyl cellobiose (pNPC) et CMC, et peu active sur la cellulose (Ohmiva et al., 1982). Cette activité est portée par une protéine de $100000 \mathrm{~d}$ pouvant exister sous forme de dimère. Une glucohydrolase active sur cellodextrine (G2 à G5) est associée aux cellules (Ohmiya et al., 1985). Elle est libérée par congélation-décongélation. Cette enzyme est importante puisqu'elle fournit directement à la bactérie du glucose à partir de polymères.

Chez $R$. flavefaciens une cellobiohydrolase très active sur pNPC mais peu sur la cellulose est également présente dans le surnageant de culture sur cellulose au bout de 7 jours (Gardner, Doerner et White, 1987). Chez B. succinogenes cultivée sur cellulose, une cellobiohydrolase périplasmique dégrade spécifiquement les cellodextrines; elle est sans activité sur le cellobiose, la cellulose ou la CMC (Huang et Forsberg, 1987). Une activité semblable également périplasmique a été retrouvée chez cette bactérie cultivée en présence de glucose pendant la phase exponentielle de croissance (Gaudet, 1987a). Sa présence en culture sur glucose semble indiquer une absence de répression catabolique. Cette cellodextrinase est spécifique des liaisons $\beta-1-4$ mais n'hydrolyse pas de polymères inférieurs à 3 unités osidiques (Huang et Forsberg, 1987). Son poids moléculaire est voisin de $40000 \mathrm{~d}$. 
Autres activités.

En général les bactéries cellulolytiques du rumen présentent également des activités hémicellulolytiques, xylanases et lichénase (Williams et Whiters, $1981 \mathrm{a}$, b, 1982 ; Williams, Whiters et Coleman, 1984). Chez $B$. succinogenes une activité xylanase est libérée par action de la trypsine sur des fractions membranaires extracellulaires (Schellhorn et Forsberg, 1984).

Un gène codant pour une xylanase a été cloné à partir de $B$. succinogenes, chez $E$. coli. Cette xylanase présente une activité spécifique équivalente à l'activité spécifique de l'enzyme chez $B$. succinogenes. Cette activité ne semble pas inductible, ni soumise à répression catabolique (Sipat et al., 1987).

\section{Genèse et conservation de l'énergie.}

Chez les bactéries anaérobies du rumen qui fermentent les sucres, I'ATP est essentiellement formé dans le cytoplasme par des phosphorylations au niveau du substrat ( Substrate level phosphorylation» ou SLP). Cependant, chez les bactéries du rumen qui présentent un taux de croissance élevé par rapport à la quantité de substrat consommé, I'ATP pourrait être également synthétisée par la voie des phosphorylations oxydatives ("Electron transfert phosphorylation " ou ETP) (Erfle, Sauer et Mahadevan, 1986; Kröger, 1978). Dans ce cas, I'ATP est synthétisée par une ATP synthétase membranaire, par l'intermédiaire d'un gradient de protons.

Les bactéries pouvant utiliser les 2 voies de synthèse d'ATP (SLP et ETP) ont un avantage certain du point de vue énergétique par rapport à celles n'utilisant que la voie SLP. Des transporteurs potentiels d'électrons (cytochromes b, flavoprotéines) et de protons (ménaquinone) ont été mis en évidence chez B. succinogenes (Dawson, Preziosi, Caldwell, 1979), et une activité fumarate-réductase a été mesurée chez $B$. succinogenes et $R$. flavefaciens (Joyner et Baldwin, 1966 ; Hopgood et Walker, 1969). Il peut donc être proposé que ces 2 bactéries cellulolytiques qui présentent par ailleurs les mêmes voies métaboliques de production de succinate, acétate et formiate, possèdent une voie ETP à fumarate-réductase. Chez $B$. succinogenes, une chaîne de transfert d'électrons à fumarate-réductase, est capable de produire un gradient de potentiel électrochimique pour les protons, parallèlement à une ATPase protonique (Taillandier et Forano, résultats non publiés). Par contre, chez $R$. albus aucune évidence d'une voie ETP n'a été rapportée.

Chez B. succinogenes, il apparaît qu'à $\mathrm{pH}$ 6,5 la force protomotrice a une valeur de $140 \mathrm{mV}$, comparable à celle obtenue chez d'autres bactéries du rumen possédant une voie ETP: Bacteroides amylophilus et Wolinella succinogenes (Mell, Wellnitz et Kröger, 1986 ; Wetzstein, McCarty et Gottschalk, 1987). Les mesures des deux composants de la force protomotrice : potentiel électrique de membrane et gradient de $\mathrm{pH}$ ont par ailleurs été réalisées pour des $\mathrm{pH}$ externes 
variant de 5,0 à 7,5 : le potentiel électrique de membrane varie de $0 \mathrm{mV}$ à $\mathrm{pH} 5,0$ à une valeur maximale de $140 \mathrm{mV}$ à pH 7,0. Les mesures de $\mathrm{pH}$ font apparaître que $B$. succinogenes régule son $\mathrm{pH}$ intracellulaire à une valeur proche de 6,5 (Taillandier et Forano, 1987), sauf pour des $\mathrm{pH}$ externes inférieurs à 5,5 où le $\mathrm{pH}$ cytoplasmique chute jusqu'à 6,0 (Russel, 1987). En conséquence de la variation de ces 2 composantes avec le $\mathrm{pH}$ externe, il apparaît que la force protomotrice chute pour des pH inférieurs à 5,5 ou supérieurs à 7,0, suggérant qu'en dehors de la zone 5,5-7,0, les mécanismes sous dépendance de la force protomotrice sont ralentis. Les chutes de la force protomotrice et du $\mathrm{pH}$ cytoplasmique à $\mathrm{pH} 5,5$ pourraient en partie expliquer les faibles taux de croissance et d'activité cellulolytique de $B$. succinogenes à ces $\mathrm{pH}$ acides (Russel et Dombrowski, 1980 ; Russel, 1987). Une propriété remarquable, est l'effet activateur des ions $\mathrm{Na}^{+}$sur la force protomotrice chez $B$. succinogenes : la carence en sodium provoque $70 \%$ d'inhibition du potentiel électrique de membrane à $\mathrm{pH} 6,7$ et une inhibition totale du gradient de $\mathrm{pH}$ à tous les $\mathrm{pH}$ considérés (Taillandier et Forano, 1987). Une activation par les ions $\mathrm{Na}^{+}$a également été mise en évidence pour le transport du glucose chez B. succinogenes (Franklund et Glass, 1987). Ces auteurs ont de plus montré que l'accumulation de glucose ne présentait pas les caractéristiques d'un système de phosphotransférase, généralement rencontré pour le transport des hexoses, mais était par contre sensible à l'ensemble des molécules dont l'action conduit à l'annulation de la force protomotrice. Ces résultats suggèrent l'existence d'un mécanisme de co-transport glucose- $\mathrm{H}^{+}$ou glucose- $\mathrm{Na}^{+}$, bien que le gradient transmembranaire $\mathrm{de} \mathrm{Na}^{+}\left(\mathrm{Na}^{+}{ }^{+}\right.$int/ $\mathrm{Nat}^{+}$ext) soit plutôt défavorable à un tel mécanisme (Forano, 1988). L'activation de l'accumulation de glucose par les ions $\mathrm{Na}^{+}$pourrait en fait être la conséquence de l'effet activateur de cet ion sur la force protomotrice. L'effet activateur du $\mathrm{Na}^{+}$sur la genèse de la force protomotrice et le transport du glucose pourrait expliquer la dépendance de B. succinogenes vis-à-vis du $\mathrm{Na}^{+}$pour sa croissance (Caldwell et Arcand, 1974).

\section{Sensibilité des bactéries cellulolytiques aux additifs alimentaires.}

Les additifs alimentaires antimicrobiens utilisés pour orienter les fermentations du rumen dans un sens bénéfique pour l'animal modifient la composition de la microflore du rumen. Les antibiotiques ionophores notamment inhibent les bactéries à Gram positif (Chen et Wolin, 1979; Dennis, Nagaraja et Bartley, 1981; Henderson, Stewart et Nekrep, 1981 ; Stewart, Grossley et Garrow, 1983); leur effet est plus marqué que celui des composés non ionophores (Nagaraja et Taylor, 1987). Cette activité anti-microbienne est due à une modification de la perméabilité membranaire spécifiquement pour le passage de certains cations. Les antibiotiques apparemment les plus actifs dans le rumen appartiennent au type "polyether". IIs ont la capacité de former des complexes liposolubles qui servent de véhicules à une large variété de cations pour traverser la membrane (voir, revue de Durand, 1982). In vivo, l'avoparcine n'affecte pas le nombre total de bactéries cellulolytiques mais réduit considérablement celui de Ruminococcus au profit de B. succinogenes (Stewart et Duncan, 1985). In vitro, 
Ruminococcus est également plus sensible à l'avoparcine que $B$. succinogenes. A l'exception de $R$. flavefaciens (souche 007) les espèces cellulolytiques cultivées sur cellulose s'adaptent à l'avoparcine (Stewart, Grossley et Garrow, 1983). Les croissances de $R$. albus, $R$. flavefaciens et $B$. fibrisolvens sont inhibées par la monensine et le lasalocide de sodium à la concentration de $2,5 \mathrm{ug} \mathrm{ml}^{-1}$ alors que $B$. succinogenes peut supporter 10 et même $20 \mathrm{ug} \mathrm{ml}^{-1}$ après adaptation (Chen et Wolin, 1979). B. succinogenes S85, R. albus, Sy3, R. flavefaciens 007 mais surtout $B$. fibrisolvens JW11 sont sensibles à la tétranasine (Newbold et al., 1988).

\section{Sensibilité aux composés phénoliques.}

L'effet des composés phénoliques, constituants habituels des parois cellulaires des fourrages dans lesquels ils sont présents sous forme d'esters d'acides hydroxycinnamiques, sur l'activité de microorganismes cellulolytiques suscite un intérêt grandissant depuis quelques années. Les acides cinnamiques réduisent le taux de dégradation de la cellulose et des parois végétales par des populations mixtes du rumen (Jung et Sahlu, 1986 ; Varel et Jung, 1986 ; Borneman, Akin et Van Eselting, 1986; Theodorou et al., 1987). Ils sont plus actifs sous forme d'esters que sous forme libre et l'effet diffère selon leur nature chimique, les espèces bactériennes et le substrat (Jung et Sahlu, 1986).

A une concentration supérieure à $5 \mathrm{mM}$, les acides $\mathrm{p}$. coumarique et férulique inhiberaient la croissance de $B$. succinogenes, $R$. flavefaciens et $R$. albus sur des oses solubles et réduiraient l'activité cellulolytique des deux premières espèces sans affecter la troisième (Chesson, Stewart et Wallace, 1982). Par contre Varel et Jung (1986) n'observent qu'un faible ralentissement de la croissance des trois espèces en présence des acides p. coumarique et férulique. $B$. succinogenes est plus sensible, selon ces auteurs, à la vanilline et à l'acide cinnamique qui inhiberaient partiellement son attachement à la cellulose. L'acide p. coumarique et le p. hydroxybenzaldéhyde seraient toxiques à l'égard de $R$. albus, $R$. flavefaciens et $B$. fibrisolvens (Borneman et al., 1986), mais les acides syringique, p. hydroxybenzoïque et hydroxycinnamique stimuleraient la croissance de ces espèces et la dégradation du papier filtre par $R$. flavefaciens. L'ultrastructure de cette dernière espèce serait altérée par les acides $p$. hydroxybenzoïque $(10 \mathrm{mM})$ et p. coumarique ( $5 \mathrm{mM}$ ) (Borneman et al., 1986).

Les espèces cellulolytiques, notamment Butyrivibrio fibrisolvens, seraient capables de transformer, au moins partiellement les acides phénoliques en composés non toxiques (Varel et Jung, 1986). Ces résultats sont toutefois controversés (Chesson, Stewart et Wallace, 1982). La croissance et l'activité cellulolytique de $R$. albus sont stimulées par l'acide 3-phénylpropanoïque (Hungate et Stack, 1982 ; Stack et Cotta, 1986) et l'acide phényl acétique (Stack, Hungate et Opsahl, 1983). In vitro, l'acide $p$. phényl propanoïque modifie à la fois la morphologie et les cellulases de $R$. albus 8 (Stack et Hungate, 1984). En présence de cet acide, $R$. albus 8 produit des cellulases essentiellement liées à la 
cellule bactérienne, un enzyme de très haut poids moléculaire ainsi que de faibles quantités de deux enzymes de faible poids moléculaire. En l'absence de cet acide, la quantité totale de cellulase produite est supérieure mais celle-ci existe essentiellement sous forme soluble de faible poids moléculaire (Stack et Hungate, 1984).

\section{Influence des minéraux.}

L'importance des éléments minéraux sur la cellulolyse dans le rumen a été soulignée dans les revues de Durand et Kawashima (1980), Smith (1984), Guéguen, Durand et Meschy (1987), Durand et Komisarczuk (1988). Les effets de certaines supplémentations minérales sur la cellulolyse, in vivo ou in vitro, avec des populations mixtes, sont probablement le résultat d'actions multiples et complémentaires sur les caractéristiques du milieu ruminal, la croissance de certaines souches et sur des paramètres spécifiques de la cellulolyse que sont l'adhérence à la cellulose et l'activité des enzymes cellulasiques. Ces derniers points ont été récemment mis en évidence dans des études réalisées avec des souches pures de $B$. succinogenes et $R$. flavefaciens. Les besoins de croissance en phosphore et en magnésium seraient ainsi trois à cinq fois plus importants pour les bactéries à Gram positif ( $R$. flavefaciens) que pour celles à Gram négatif (B. succinogenes) (Komisarczuk et al., non publié). En outre le phosphore, le calcium et le magnésium stimuleraient l'activité endoglucanasique de B. succinogenes (Komisarczuk et al., 1987b; 1988), alors que les cations divalents $\mathrm{Ca}^{++}$et $\mathrm{Mg}^{++}$favoriseraient ensemble l'adhérence à la cellulose de $R$. flavefaciens (Roger et al., 1988). Des travaux actuellement réalisés in vitro en Rusitec sur les conséquences métaboliques de la carence en soufre, indiquent également un effet stimulateur de cet élément sur l'activité des endoglucanases de populations mixtes, dans la phase liquide associée aux particules solides (Stevani et al., 1988).

A ce jour, seul le phosphore a fait l'objet d'études pour quantifier un besoin spécifique à la dégradation de la cellulose en population mixte, supérieur au besoin de protéosynthèse (Komisarczuk et al., 1987a). De nombreux travaux restent à faire, notamment dans le cas du soufre et du magnésium.

\section{Conclusion.}

Malgré les difficultés inhérentes à la culture des espèces bactériennes strictement anaérobies, des résultats intéressants ont été apportés et plusieurs réponses concernant les mécanismes sont en cours d'ébauche. Les techniques de biologie moléculaire ont permis d'isoler et de purifier des enzymes du complexe cellulasique dont il faut encore préciser la localisation cellulaire et l'activité. Les mécanismes de dégradation de la cellulose ne sont pas pour autant élucidés car 
les activités hydrolytiques mesurées in vitro restent très inférieures aux capacités réelles des bactéries à dégrader la cellulose. L'étude des mécanismes d'adhérence qui doit rester prioritaire compte tenu de l'importance du phénomène dans le rumen connaîtra des progrès dès que l'on disposera de souches mutées et que les facteurs de l'adhésion auront été clonés. A l'image de ce qui a déjà été réalisé pour certaines espèces bactériennes du tube digestif de l'homme (Kuritza, Schauchnessy et Salyers, 1986) la fabrication de sondes d'hybridation ADN pour les espèces importantes du rumen, cellulolytiques ou non, permettra enfin de suivre in situ les flux de populations bactériennes (Stahl et al., 1988). L'acquisition de l'ensemble de ces connaissances constitue un préalable indispensable si l'on veut in fine espérer maîtriser les équilibres écologiques au sein du rumen et réussir un jour à le coloniser par des souches plus performantes, si tant est que cela soit possible.

4èmes Journées sur la Nutrition et l'Alimentation des Herbivores, I.N.R.A., Paris, 24 et 25 mars 1988.

\section{Références}

ANDERSON K. L., NAGARAJA T. G., MORRILL J. L., AVERY T. B., GALITZER S. J., BOYER J. E., 1987. Ruminal microbial development in conventionally or early weaned calves. J. anim. Sci. 64, 1215-1226.

BARROS M. E. C., THOMSON J. A., 1987. Cloning and expression in Escherichia coli of a cellulase Gene from Ruminoccocus flavefaciens. J. Bacteriol., 169, 1760-1762.

BORNEMAN W. S., AKIN D. E., VAN ESELTING W. P., 1986. Effect of phenolic monomers on ruminal bacteria. Appl. environ. Microbiol., 52, $1331-1339$.

BRYANT M.P., 1973. Nutritional requirements of the predominant rumen cellulolytic bacteria. Fed. Proceed., 32, 1809-1813.

CALDWELL D. R., ARCAND C., 1974. Inorganic and metal-organic growth requirements of the genus Bacteroides. J. Bacteriol., 120, 322-333.

CAVICCHIOLI R., WATSON K., 1987. Secretion of endoglucanase from Bacteroides succinogenes S-O cloned in E. coli. In Biochemistry and genetics of cellulose degradation, p. 70, FEMS Symp., Paris, 7-9 septembre.

CHEN M., WOLIN M.J., 1979. Effect of monensin and lasalocid-sodium on the growth of methanogenic and rumen saccharolytic bacteria. Appl. environ. Microbiol., 38, 72-77.

CHENG K. J., COSTERTON J. W., 1980. Adherent rumen bacteria, their role in the digestion of plant material, urea and epithelial cells, 227-250. In RUCKEBUSH Y., THIVEND P., Digestive physiology and metabolism in ruminants. M.T.P. Press Ltd., Lancaster, England.

CHENG K. J., STEWART C. S., DINSDALE D., COSTERTON J. W., 1984. Electron microscopy of bacteria involved in the digestion of plant cell walls. Anim. Feed Sci. Techn., 10, 93-120.

CHESSON A., STEWART C. S., DALGARNO K., KUNG T. P., 1986. Degradation of isolated grass mesophyll, epidemidis and fibre cell walls in the rumen and by cellulolytic rumen bacteria in axenic culture. J. appl. Bacteriol., 60, 327-336.

CHESSON A., STEWART C. S., WALLACE R. J., 1982. Influence of plant phenolic acids on growth and cellulolytic activity of rumen bacteria. Appl. environ. Microbiol., 44, 597-603.

DAWSON K. A., PREZIOSI M.C., CALDWELL D. R., 1979. Some effects of uncouplers and inhibitors on growth and electron transport in rumen bacteria. J. Bacteriol., 139, 384-392.

DENNIS S. M., NAGARAJA T. G., BARTLEY E. E., 1981. Effects of Lasalocid or monensin on lactate producing or using rumen bacteria. J. anim. Sci., 52, 418-426.

DURAND M., 1982. Orientation du métabolisme du rumen au moyen des additifs. Ann. Zootech., 31, 47-76. 
DURAND M., KAWASHIMA R., 1980. Influence of minerals in rumen microbial digestion, 375-408. In RUCKEBUSH Y., THIVEND P., Digestive physiology and metabolism in ruminants. M.T.P. Press Ltd, Lancaster, England.

DURAND M., KOMISARCZUK S., 1988. Minerals and rumen Microbiota. J. Nutr., 118, 249-260.

ERFLE J. D., SAUER F. D., MAHADEVAN S., 1986. Energy metabolism in rumen microbes, 81 -99. In MILLIGAN L. P., GROWUM W. L., DOBSON A., Control of digestion and metabolism in ruminants. Prentice Hall. Englewood Cliffs, U.S.A.

FONTY G., GOUET Ph., JOUANY J. P., SENAUD J., 1983. Ecological factors determining the establishment of cellulolytic bacteria and protozoa in the rumen of meroxenic lambs. $J$. gen. Microbiol., 129, 213-223.

FONTY G., GOUET Ph., JOUANY J. P., SENAUD J., 1987a. Establishment of the microflora and anaerobic fungi in the rumen of lambs. J. gen. Microbiol., 123, 1835-1843.

FONTY G., GOUET Ph., JOUANY J. P., CHAVAROT M., ROUSSEL O., 1987b. The conventional lamb placed in a sterile isolator 24 hours after birth, and animal model to study the activity of the different cellulolytic micro-organisms. In Gnotobioloby and its application. Proc. IXth Int. Symp. on Gnotobiology, Versailles, France.

FONTY G., GOUET Ph., RATIFIARIVELO H., JOUANY J. P., 1988a. Establishment of Bacteroides succinogenes and measurement of the main digestive parameters in the rumen of gnotoxenic lambs. Can. J. Microbiol., 34, 938-946.

FONTY G., GOUET Ph., NEBOUT J. M., 1988b. Development of cellulolytic microflora in the rumen of lambs transferred into sterile isolators a few ays after birth. Can. J. Microbiol. (sous presse).

FONTY G., ROGER V., GOUET Ph., 1987c. Factors affecting adhesion to cellulose of two rumen cellulolytic bacteria : Bacteroides succinogenes and Ruminoccocus flavefaciens. An in vitro study. In Biochemistry and genetics of cellulose degradation, p. 63, FEMS Symp., Paris, 7-9 septembre.

FORANO E., 1988. Effets de la monensine sur les gradients transmembranaires de $\mathrm{H}^{+}, \mathrm{Na}^{+}, \mathrm{K}^{+}$chez Bacteroides succinogenes, bactérie cellulolytique du rumen. Reprod. Nutr. Dévelop., 28, Suppl. $n^{\circ} 1$.

FRANKLUND C.V., GLASS T. L., 1987. Glucose uptake by the cellulolytic ruminal anaerobe Bacteroides succinogenes. J. Bacteriol., 169, 500-506.

GARDNER R. M., DOERNER K. C. and WHITE B. A., 1987. Purification and characterization of an Exo- $\beta$-1,4-glucanase from Ruminoccocus flavefaciens FD-1. J. Bacteriol., 169, $4581-4588$.

GAUDET G., 1983. Les cellulases extracellulaires de Bacteroides succinogenes. Ann. Microbiol. (Inst. Pasteur), 134a, 111-114.

GAUDET G., 1987a. Les produits de dégradation de la cellulose par les cellulases de Bacteroides succinogenes, bactérie cellulolytique du rumen. Reprod. Nutr. Dévelop., 27, 239-240.

GAUDET G., 1987b. Bacteroides succinogenes cellular location of cellulolytic enzymes. In Biochemistry and genetics of cellulose degradation, p. 62. FEMS Symp., Paris, 7-9 septembre.

GAUDET G., GAILLARD B., 1987. Vesicle formation and cellulose degradation in Bacteroides succinogenes cultures: ultrastructural aspects. Arch. Microbiol., 148, 150-154.

GOUET Ph., FONTY G., JOUANY J.P., NEBOUT M., 1984. Cellulolytic bacteria and rumen digestion in lambs isolated after birth. Can. J. anim. Sci., 64, (suppl.), 163-163.

GRAHAM H., AMAN P., THEAUDER O., KOLYANKAYA N., STEWART C. S., 1985 . Influence of heat sterilization and ammoniation on straw composition and degradation by pure cultures of cellulolytic rumen bacteria. Anim. Feed. Sci. Technol. 12, 195-203.

GROLEAU D., FORSBERG C. W., 1983a. Partial characterization of the extracellular carboxymethylcellulase activity produced by the rumen bacterium Bacteroides succinogenes. Can. $J$. Microbiol., 29, 504-517.

GROLEAU D., FORSBERG C. W., 1983b. Solubilization of the extracellular membraneous carboxymethylcellulase of Bacteroides succinogenes by trypsin. Can. J. Microbiol., 29, 710-717.

GUÉGUEN L., DURAND M., MESCHY F., 1987. Apports recommandés en éléments minéraux pour les ruminants. Bull. Techn. CRZV -Theix, 70, 105-112.

HENDERSON C., STEWART C. S., NEKREP F. V., 1981. The effect of monensin on pure and mixed cultures of rumen bacteria. J. appl. Bacteriol., 51, 159-169. 
HOBSON P. N., MANN S. O., STEWART C. S., 1981. Growth and rumen function of gnotobiotic lambs fed on starchy diets. J. Gen. Microbiol., 126, 219-230.

HOPGOOD M. F., WALKER D. J., 1969. Succinic acid production by rumen bacteria. III. Enzymic studies on the formation of succinate by Ruminococcus flavefaciens. Aust. J. biol. Sci., 22. $1413-1424$

HUANG C. M., KELLY W. J., CURRY M. M., YU P. L., ASMUNDSON R. V., 1987. Purification and cloning of cellulase from Ruminococcus flavefaciens. Biochemistry and genetics of cellulose degradation, p. 70, FEMS, Symp., Paris, $7-9$ septembre.

HUANG L., FORSBERG C. W., 1987. Isolation of a cellodextrinase from Bacteroides succinogenes. Appl. environ. Microbial., 53, 1034-1041.

HUNGATE R. E., 1950. The anaerobic mesophilic cellulolytic bacteria. Bacteria. Rev., 14, 1-47

HUNGATE R. E., 1966. The rumen and its microbes. Acad. Press. New York.

HUNGATE R. E., STACK R. J., 1982. Phenyl propanoic acid: growth factor for Ruminococcus albus. Appl, environ. Microbiol., 44, 79-83

JOYNER A., BALDWIN R. L., 1966. Enzymatic studies of pure culture of rumen micro-organisms. J. Bacteriol., 92, $1321-1330$.

JUNG H.I. G., SAHLU T., 1986. Depression of cellulose digestion by esterified phenolic acids. J. Food. Sci. Agric., 37, 659-665.

KAWAI S., HONDA H., TANASE T., TAYA M., IIJIMA S., KOBAYASHI T., 1987. Molecular cloning of Ruminoccocus albus. Cellulase. Gene. Agric. biol. Chem., 51, 59-63.

KOLANKAYA N., STEWART C. S., DUNCAN S. H., CHENG K. J., COSTERTON J. W., 1985. The effect of ammonia treatment on the solubilization of straw and the growth of cellulolytic bacteria. J. appl. Bacteriol., 58, 371-379.

KOMISARCZUK S., MCALLAN A. B., MERRY R. J., 1987a. Effect of different levels of phosphorous on rumen microbial fermentation and synthesis determined using a continuous culture technique. Br. J. Nutr., 57, 279-290.

KOMISARCZUK S., FONTY G., ROGER V., GAUDET G., 1987b. Effect of some major minerals on growth and cellulolytic activity of Bacteroides succinogenes. In Biochemistry and genetics of cellulose degradation, p. 57, FEMS, Symp., Paris, 7-9 septembre.

KOMISARCZUK S., GAUDET G., HANNEQUART G., FONTY G., DURAND M., 1988 . Effets d'une sub-carence en phosphore sur divers paramètres d'activité de Bacteroides succinogenes. Reprod. Nutr. Dévelop., 28, Suppl. $\mathrm{n}^{\circ} 1$.

KUDO H., CHENG K.J., COSTERTON J.W., 1987. Electron microscopy of the methylcellulose-mediated detachment of cellulolytic rumen bacteria from cellulose fibers. Can. J. Microbiol., 33, 267-272.

KURITZA A. P., SHAUGHNESSY P., SALYERS A. A., 1986. Enumeration of polysaccharide degrading Bacteroides species in human feces by using specific DNA probes. Appl. environ. Microbiol., 51, 385-380

KRÖGER A., 1978. Fumarate as terminal acceptor of phosphorylative electron transport. Biochim Biophys. Acta, 505, 129-145.

LAMED R., NAIMARK J., MORGENSTEIN E., BAYER E. A., 1987. Specialized cell surface structures in cellulolytic bacteria. J. Bacteriol., 169, 3792-3800.

LYSONS R. J., ALEXANDER T. J. L., WELLSTEAD P., HOBSON P. N., MANN S. O., STEWART C. S., 1976. Defined bacterial populations in the rumen of gnotobiotic lambs. J. gen. Microbiol., 94, 257-269.

MANN S. O., STEWART C. S., 1974. Establishment of a limited flora in gnotobiotic lambs fed on a roughage diets. J. gen. Microbiol., 84, 379-382.

MELL H., WELLNITZ C., KROGER A., 1986. The electrochemical proton potential and the proton/electron ratio of the electron transport with fumarate in Wolinella succinogenes. Biochim. Biophys. Acta, 852, 212-221.

MONTGOMERY L., FLESHER B., STAHL D., 1988. Transfer of Bacteroides succinogenes (Hungate) to Fibrobacter gen. nov. as Fibrobacter succinogenes comb. nov. and Description of Fibrobacter intestinalis sp. nov. Int. J. Syst. Bacteriol., 38, 430-435.

MORRIS E. J., 1987. Characteristics of the adhesion of Ruminococcus albus to cellulose. FEMS Microbiol. Lett., 51, 113-118. 
MORRIS E. J., VAN GYLSWYK N. O., 1980. Comparison of the action of rumen bacteria on cell walls from Eragrostis Tel. J agric. Sci. Camb., 95, 313-323

NAGARAJA J. G., TAYLOR M. B., 1987. Susceptibility and resistance of a ruminal bacteria to antimicrobial feed additives. Appl. environ. Microbiol., 53, 1620-1625.

NEWBOLD C. J., WALLACE R. J., WATT N. D., RICHARDSON A. J., 1988. Effect of the novel ionophore tetronasin (ICl 139603) on ruminal micro-organisms. Appl. environ. Microbiol., 54, 544-547.

OHMIYA K., MASATOSHI S., TAYA M., SHOICHI S., 1982. Purification and properties of a cellobiosidase from Ruminococcus albus. J. Bacteriol, 150, 407-409.

OHMIYA K., SHIRAIM., KURACHIY., SHIMIZU S., 1985. Isolation and properties of $\beta$-glucosidase from Ruminoccocus albus. J. Bacteriol., 161, 432-434.

ROGER V., FONTY G., KOMISARCZUK S., GOUET Ph., 1988. Effet de quelques facteurs physico-chimiques sur l'adhésion à la cellulose de deux espèces bactériennes cellulolytiques du rumen. Reprod. Nutr. Dévelop., 28, Suppl. $\mathrm{n}^{\circ} 1$.

RUSSELL J. B., 1987. Effect of extracellular $\mathrm{pH}$ on growth and proton motive force of Bacteroides succinogenes, a cellulolytic ruminal bacterium. Appl. environ. Microbiol., 53, 2379-2383.

RUSSELL J. B., DOMBROWSKI D. B., 1980. Effect of $\mathrm{pH}$ on the efficiency of growth by pure cultures of rumen bacteria in continuous culture. Appl environ. Microbiol., 39, 604-610.

SCHELLHORN H. E., FORSBERG C. W., 1984. Multiplicity of extracellular $\beta-(1,4)$-endoglucanase of Bacteroides succinogenes S85. Can. J. Microbiol., 30, 930-937.

SMITH R. H., 1984. Minerals and rumen function. In Nuclear techniques in tropical animal diseases and nutritional desorders, pp. 79-96. International Atomic Energy Agency. Vienna, Austria.

SIPAT A., TAYLOR K. A., LO R. Y. C., FORSBERG C. W., KRELL P. J., $1987 . \quad$ Molecular cloning of a xylanase gene from Bacteroides succinogenes and its Expression in Escherichia coli. Appl. environ. Microbiol., 53, 477-481.

STACK R. J., COTTA M. A., 1986. Effect of 3-phenyl propanoic acid on growth and cellulose utilization by cellulolytic ruminal bacteria. Appl environ. Microbiol., 52, 209-210.

STACK R. J., HUNGATE R. E., 1984. Effect of 3-phenyl propanoic acid on capsule and cellulases of Ruminococcus albus 8. Appl. environ. Microbiol., 48, 218-223.

STACK R. J., HUNGATE R. E., OPSAHL W.P., 1983. Phenylacetic acid stimulation of cellulose digestion by Ruminoccocus albus 8. Appl. environ. Microbiol., 46, 339-544.

STAHL D. A., FLESHER B. A., MANSFIELD H. R., MONTGOMERY L., 1988. Use of phyllogenetically based hybridization probes for studies of ruminal microbial ecology. Appl. environ. Microbiol., 54, 1079-1084.

STEVANI J., BEAUMARTIN Ph., DUMAY Ch., HANNEQUART G., DURAND M., $1988 . \quad$ Etude en fermenteur semi-continu de l'influence de l'addition de sulfate sur la dégradation des constituants pariétaux d'une paille traitée ou non à la soude. Reprod. Nutr. Dévelop., 28, Suppl. $\mathbf{n}^{\circ} 1$

STEWART C. S., 1981. Rumen microbiology. In DOWSCH K. H., FERRANTI M. P., THEANDER O., Improved utilization of lignocellulosic materials for animal feed. Proc. OECD/COST Workshop. Braunschweig, sept. 30-oct. 2.

STEWART C. S., DUNCAN S. H., 1985. The effect of Avoparcin on cellulolytic bacteria of the bovine rumen. J. gen. Microbiol., 31, 427-435.

STEWART C. S., GROSSLEY M. V., GARROW S. H., 1983. The effect of avoparcin on laboratory cultures of rumen bacteria. Eur. J. appl. Microbiol. Biotechnol., 17, 292-297.

TAILLANDIER D., FORANO E., 1987. The proton electrochemical gradient in Bacteroides succinogenes, a rumen cellulolytic bacterium. In Biochemistry and genetics of cellulose degradation, p. 62. FEMS, Symp., Paris, 7-9 septembre.

TAYLOR K. A., CROSBY B., McGAVIN M., FORSBERG C. W., THOMAS D. Y., 1987. Characteristics of the endoglucanase encoded by a celgene from Bacteroides succinogenes expressed in Escherichia coli. Appl. environ. Microbiol., 53, 41-46.

THEODOROU M. K., GASCOYNE D. I., AKIN D. E., HARTLEY R. D., $1987 . \quad$ Effect of phenolic acids and phenolics from plant cell walls on rumen like fermentation in consecutive batch culture. Appl. environ. Microbiol., 53, $1046-1050$.

VAREL U. H., JUNG H. S., 1986. Influence of forage phenolics on ruminal fibrolytic bacteria and in vitro fiber degradation. Appl. environ. Microbiol., 52, 275-280. 
WETZSTEIN H. G., MCCARTHY J. E. G., GOTTSCHALK G., 1987. The membrane potential in a cytochrome-deficient species of Bacteroides : its magnitude and mode of generation. J. gen. Microbiol., 133, 73-83.

WILLIAMS A. G., WITHERS S. E., 1981a. Hemicellulose-degrading enzymes synthesized by rumen bacteria. J. appl. Bacteriol., 51, 375-385.

WILLIAMS A. G., WITHERS S. E., $1981 \mathrm{~b}$. The production of plant cell wall polysaccharide-degrading enzymes by hemicellulolytic rumen bacterial isolates grown on a range of carbohydrate substrates. J. appl. Bacteriol., 52, 377-387.

WILLIAMS A. G., WITHERS S. E., 1982. The effect of the carbohydrate growth substrate on the glycosidase activity of hemicellulose-degrading rumen bacterial isolates. J. appl. Bacteriol., 52. 389-401.

WILLIAMS A. G., WITHERS S. E., COLEMAN G. S., 1984. Glycoside hydrolases of rumen bacteria and protozoa. Curr. Microbiol., 10, 287-294.

WOOD T. M., WILSON C. A., STEWART C. S., 1982. Preparation of the cellulase from the cellulolytic anaerobic rumen bacterium Ruminococcus albus and its release from the bacterial cell wall. Biochem. J., 205, 129-137. 